\title{
MAPK and Hippo signaling pathways crosstalk via the RAF-1/MST-2 interaction in malignant melanoma
}

\author{
RUIZHENG FENG ${ }^{1}$, JUNSHENG GONG $^{1}$, LINA WU $^{2}$, LEI WANG $^{3}$, BAOLIN ZHANG ${ }^{1}$, \\ GANG LIANG $^{2}$, HUIXIA ZHENG ${ }^{2}$ and HONG XIAO ${ }^{2}$
}

Departments of ${ }^{1}$ Plastic Surgery and ${ }^{2}$ Pathology, The First Hospital of Shanxi Medical University, Taiyuan, Shanxi 030024; ${ }^{3}$ Department of Gerontology, Shanxi Dayi Hospital, Taiyuan, Shanxi 030000, P.R. China

Received November 11, 2016; Accepted June 14, 2017

DOI: $10.3892 / o r .2017 .5774$

\begin{abstract}
The aim of the present study was to expound on the interactions between the mitogen-activated protein kinase (MAPK) and Hippo pathway members, and to further elucidate the molecular mechanisms of melanoma tumorigenesis. Four melanoma cell lines (C32, HS695T, SK-MEL-28 and A375) were used in the present study. Western blotting was used to assess the expression levels of the MAPK and Hippo pathway effector proteins: rapidly accelerated fibrosarcoma-1 proto-oncogene, serine/threonine kinase (RAF-1); serine/threonine kinase 3 (STK3; also known as MST-2); yes-associated protein (YAP); and tafazzin (TAZ). Immunoprecipitation was used to identify interactions between the effector proteins of the Hippo and MAPK pathways. RAF-1 was knocked down in melanoma cells using siRNA transfection, and cell proliferation, migration and invasion were determined by the MTT, wound-healing and Transwell invasion assays, respectively. Additionally, the cell cycle and apoptosis were analyzed by flow cytometry $48 \mathrm{~h}$ after RAF-1 knockdown. We found that the expression levels of the four proteins were variable, and that the HS695T cells expressed the highest levels of RAF-1. Immunoprecipitation studies revealed that RAF-1 bound to MST-2 in melanoma cells. Knockdown of RAF-1 inhibited the expression of YAP and TAZ, but did not affect MST-2 expression. Additionally, RAF-1 knockdown in melanoma cells significantly inhibited cell proliferation, migration and invasion, and induced apoptosis in these cells. Collectively, our results indicate that the RAF-1/MST-2 interaction may be a novel link between the MAPK and Hippo pathways.
\end{abstract}

\section{Introduction}

Melanoma is the most dangerous type of skin tumor, although it accounts for less than $5 \%$ of all skin tumors. It is respon-

Correspondence to: Dr Ruizheng Feng, Department of Plastic Surgery, The First Affiliated Hospital, Shanxi Medical University, 85 South Jiefang Road, Taiyuan, Shanxi 030024, P.R. China

E-mail: tingyao38y@163.com

Key words: melanoma, RAF-1, MST-2, MAPK pathway, Hippo pathway sible for over $80 \%$ of all skin cancer-related deaths. In 2012 , 232,000 new cases of melanoma and 55,000 melanoma-related deaths were reported worldwide (1). Moreover, the incidence of melanoma is increasing at a rate faster than that of any other solid tumor, and is thought to be the highest in white-skinned people living at low latitudes (2). In its advanced stages, melanoma is highly malignant, owing to its potential for distant metastasis (3), and an extremely low 5-year survival rate $(5-16 \%)$ (4). Unfortunately, melanoma is refractory to conventional chemotherapeutics, thus, the treatment options for patients with advanced disease are limited (5). Understanding the molecular mechanisms of melanoma may help to improve the current therapeutic strategies.

The mitogen-activated protein kinase (MAPK) signaling pathway is a key regulator of cellular growth and proliferation, and has been found to play crucial roles in the pathogenesis of melanoma. Most melanomas exhibit constitutive activation of the MAPK pathway (6). Mutations in the rapidly accelerated fibrosarcoma isoform B (BRAF) lead to constitutive activation of the MAPK pathway and are associated with poor outcome in melanoma $(7,8)$. It has been reported that more than half of the melanoma cases contain BRAF mutations (9). Thus, a distinct approach may be to use BRAF inhibitors to extend survival in patients with metastatic melanoma (10). However, preexisting or acquired resistance to these agents appears soon following a transient response (11). In addition, various studies have found that BRAF inhibitors may cause acanthopapilloma, keratoacanthoma or cutaneous squamous-cell carcinoma in the early stages of treatment $(11,12)$. Therefore, identification of additional core members of key molecular pathways implicated in the pathogenesis of melanoma is crucial for the design of novel therapies.

The RAF family of serine/threonine kinases is comprised of three members: CRAF (RAF-1), BRAF and ARAF $(13,14)$. As aforementioned, BRAF is reportedly involved in the pathogenesis of melanoma via the MAPK pathway. Notably, RAF-1 was also found to play an important role in the activation of the MAPK pathway in melanoma $(15,16)$. RAF-1 dysregulation represents a prominent resistance mechanism in melanoma (14,17). Furthermore, RAF-1 can bind to serine/ threonine kinase 3 (STK3), also known as MST-2, thus influencing apoptosis $(18,19)$. Notably, MST-2 is one of the core components of the Hippo pathway in mammals, which is 
involved in cell proliferation, growth, and apoptosis $(17,20)$. Alterations in the Hippo pathway have been found to be associated with tumorigenesis, including melanoma development $(21,22)$. However MST-2, yes-associated protein (YAP) and tafazzin (TAZ) are also major effectors of the Hippo pathway, and have been suggested to contribute to the metastatic and invasive capacities of melanoma cells (23). Moreover, it has been demonstrated that YAP can regulate the response of cancer cells to MAPK pathway inhibitors (24). Tumorigenesis is a consequence of the combined action of many factors and intricate pathways. In light of the role of the MAPK and Hippo pathways and their effectors (RAF-1, MST-2, YAP and TAZ) in melanoma, we speculated that there may exist some links among these pathways via their effectors in melanoma tumorigenesis.

In the present study, we assessed the expression levels of RAF-1, MST-2, YAP and TAZ proteins of the MAPK and Hippo pathways in four melanoma cell lines and found that RAF-1 formed a complex with MST-2. We further investigated the effects of RAF-1/MST-2 interaction on melanoma cell proliferation, migration and invasion, as well as on the cell cycle and apoptosis of melanoma cells. The present study, expounds on the interactions between the MAPK and Hippo pathway members elucidating the molecular mechanisms of melanoma tumorigenesis.

\section{Materials and methods}

Cell culture. Four melanoma cell lines, including C32, HS695T, SK-MEL-28 and A375, were used in the present study. C32, HS695T and SK-MEL-28 were cultured in minimum essential medium (MEM). A375 was cultured in Dulbecco's modified Eagle's medium (DMEM) containing $100 \mathrm{U} / \mathrm{ml}$ penicillin, $10 \%$ fetal bovine serum (FBS), and $100 \mu \mathrm{g} / \mathrm{ml}$ streptomycin. The cell lines were maintained in a humid atmosphere at $37^{\circ} \mathrm{C}$ and $5 \% \mathrm{CO}_{2}$.

Western blot analysis. After being washed with ice-cold phosphate-buffered saline (PBS), the four cell lines were lysed in whole-cell extraction buffer. Proteins $(20 \mu \mathrm{l})$ were resolved using $10 \%$ sodium dodecyl sulfate (SDS)-polyacrylamide gel electrophoresis (PAGE), and transferred to polyvinylidene fluoride (PVDF) membranes. The membranes were blocked with $5 \%$ skim milk and incubated overnight at $4^{\circ} \mathrm{C}$ with primary antibodies specific to human RAF-1, YAP and TAZ (sc-227, sc-15407 and sc-17130, respectively; 1:1,000; Santa Cruz Biotechnology, Santa Cruz, CA, USA); MST-2 (\#3952; 1:1,000; Cell Signaling Technology, Boston, MA, USA); and glyceraldehyde 3-phosphate dehydrogenase (GAPDH; 1:1,000; Santa Cruz Biotechnology). Then, the membranes were incubated with the appropriate secondary antibodies for $2 \mathrm{~h}$ at $25^{\circ} \mathrm{C}$. The membranes were washed and the protein bands were visualized using 3,3'-diaminobenzidine (DAB).

Immunoprecipitation. Cells were seeded in 6-well plates and lysed with ice-cold radioimmunoprecipitation assay (RIPA) buffer. Homogenates were precleared with $20 \mu \mathrm{l}$ of Protein A-Agarose (Santa Cruz Biotechnology) at $4^{\circ} \mathrm{C}$ for $1 \mathrm{~h}$ with rocking. RAF-1 proteins were added as bait and immunoprecipitated with MST- 2 and incubated overnight at $4^{\circ} \mathrm{C}$ with rocking. The bound proteins were then immunoprecipitated with Protein A-Agarose for $2 \mathrm{~h}$ at $4^{\circ} \mathrm{C}$. After being washed, the immune complexes were released in $15 \mu 1$ of $2 \mathrm{X}$ SDS loading buffer by boiling at $100^{\circ} \mathrm{C}$ for $5 \mathrm{~min}$ and resolved by SDS-PAGE. The immune complexes were then transferred to PVDF membranes and subjected to immunoblot analysis.

RAF-1 knockdown. Cells were seeded in plates $24 \mathrm{~h}$ before transfection. RAF-1-siRNA and negative control siRNAs (Santa Cruz Biotechnology) were transfected using Lipofectamine 2000 according to the manufacturer's protocol. The efficacy and specificity of RAF-1 knockdown were ascertained by western blot analysis.

Cell viability assay. The cell viability after RAF-1 knockdown was determined by the 3-(4,5-dimethylthiazol-2-yl)-2,5-diphenyltetrazolium bromide (MTT) assay. Transfected cells were cultured in a 96-well plate for $48 \mathrm{~h}$ and $20 \mu \mathrm{l}$ of MTT was added to each well. After incubation for $4 \mathrm{~h}, 150 \mu \mathrm{l}$ of dimethyl sulfoxide (DMSO) was added and the optical density (OD) at $490 \mathrm{~nm}$ was assessed. The inhibitory rate (IR) was calculated as follows:

Inhibitory rate (IR) $(\%)=\left[1-\left(\mathrm{OD}_{\text {transfection }} / \mathrm{OD}_{\text {control }}\right)\right] \times 100 \%$

Cell migration assay. Wound-healing assay was used to detect the effects of RAF-1-siRNA on the migration ability of cells. Cells in three groups (RAF-1-siRNA, control-siRNA and no-siRNA control) were cultured in 6-well plates. After the cells reached $90 \%$ confluence, a wound track, $\sim 5 \mathrm{~mm}$ in size, was scored in each dish. After $48 \mathrm{~h}$, the cells that migrated into the wounded area were visualized and photographed. The healing rate (HR) was calculated as follows:

Healing rate $(\mathrm{HR})(\%)=[1-($ scratch area at each time-point $/$ scratch area at time 0$)] \times 100 \%$

Cell invasion assay. The invasion assay was performed using Transwell cell culture chambers as previously described (25). After $48 \mathrm{~h}$ of transfection, the cells were harvested with $0.02 \%$ ethylenediaminetetraacetic acid (EDTA), and suspended in serum-free MEM. Then, $100 \mu \mathrm{l}$ of cell suspension at a density of $2 \times 10^{5}$ cells $/ \mathrm{ml}$ was added to the upper chamber and MEM containing $20 \%$ FBS was added to the lower compartment of the Transwell. The chambers were incubated at $37^{\circ} \mathrm{C}$ for $4 \mathrm{~h}$, and the cells on the upper surface of the filter, which had not migrated, were removed with cotton swabs. The migrated cells on the lower surface of the filter were collected, fixed with methanol, and stained with hematoxylin. Cells in $>10$ random fields of view were counted at a magnification of $\times 200$, under an inverted microscope.

Cell cycle assay. The cell cycle analysis of transfected cells was performed using flow cytometry. Transfected cells were seeded into a $6-\mathrm{cm}$ dish at a density of $2 \times 10^{5} \mathrm{cells} / \mathrm{ml}$, and cultured in a $37^{\circ} \mathrm{C}$ incubator for $40 \mathrm{~h}$. After being washed with ice-cold PBS, the cells were fixed with ice-cold $70 \%$ ethanol for $30 \mathrm{~min}$ at $4^{\circ} \mathrm{C}$, and then resuspended in PBS. The suspension was filtered and stained with propidium iodide (PI; BD Biosciences, San Jose, CA, USA) for $30 \mathrm{~min}$ at $4^{\circ} \mathrm{C}$ in the 

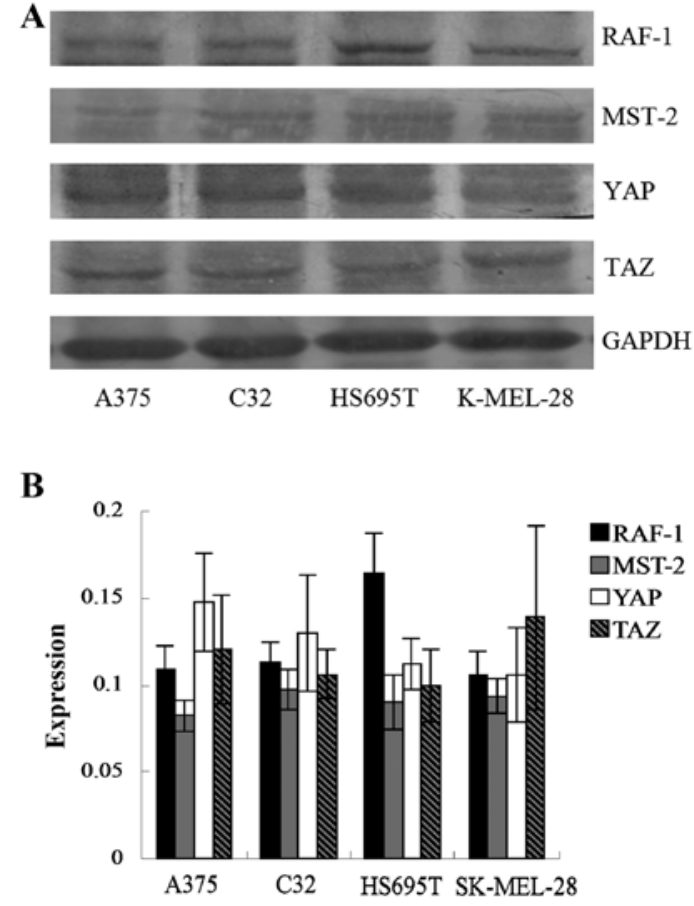

Figure 1. Protein expression levels of MAPK and Hippo pathway members in human melanoma cell lines. (A) Western blotting showing RAF-1, MST-2, YAP and TAZ expression in four human melanoma cell lines. (B) Quantification of RAF-1, MST-2, YAP and TAZ expression levels relative to GAPDH. RAF-1, fibrosarcoma-1 proto-oncogene, serine/threonine kinase; MST-2, serine/threonine kinase 3; YAP, yes-associated protein; TAZ, tafazzin.

dark. The DNA content was analyzed using flow cytometry. Three independent experiments were performed.

Cell apoptosis assay. The transfected cells were seeded in a culture dish at a density of $2 \times 10^{5}$ cells $/ \mathrm{ml}$, and cultured at $37^{\circ} \mathrm{C}$ in an incubator for $40 \mathrm{~h}$. After being washed twice with ice-cold PBS, the cells were resuspended in $1 \mathrm{X}$ binding buffer (BD Biosciences). Apoptosis of the transfected cells was quantified by staining with $5 \mu 1$ of Annexin-fluorescein isothiocyanate (FITC; BD Biosciences) and $2.5 \mu \mathrm{l}$ of PI. The cells were incubated on ice for $10 \mathrm{~min}$ in the dark and $400 \mu \mathrm{l}$ of $1 \mathrm{X}$ binding buffer was added. The apoptosis rates were analyzed using flow cytometry.

Statistical analysis. Statistical analyses were performed using SPSS 15.0 (SPSS, Inc., Chicago, IL, USA). Data are presented as the mean \pm standard deviation (SD) from at least three independent experiments. Data were analyzed by Student's t-test (for two groups) or one-way analysis of variance (ANOVA; for three or more groups). $\mathrm{P}<0.05$ was considered to indicate a statistically significant result.

\section{Results}

Expression of MAPK and Hippo pathway members in human melanoma cell lines. The expression levels of the MAPK and Hippo pathway effector proteins, RAF-1, MST-2, YAP and TAZ, in four human melanoma cell lines were assessed by western blotting. As shown in Fig. 1A and B, the expression levels of these proteins were variable across the panel. HS695T

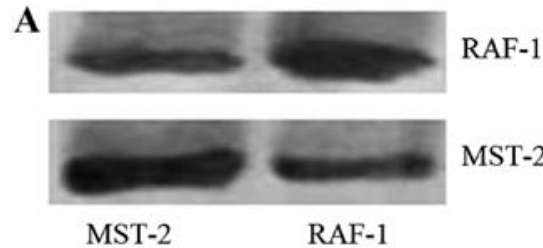

B
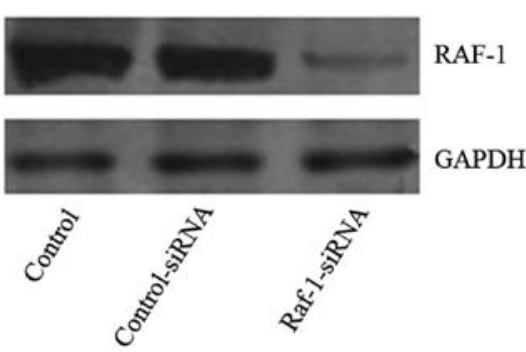

C

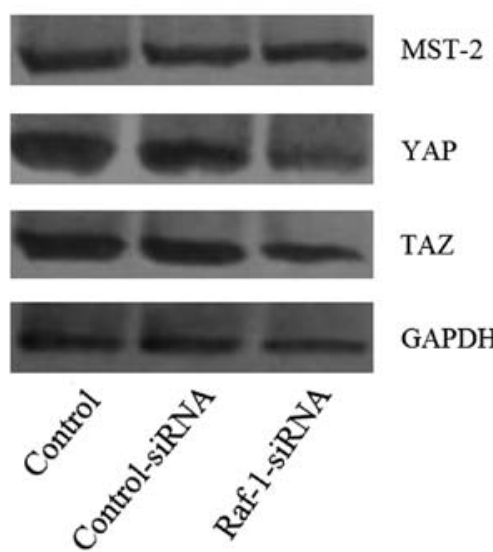

Figure 2. Link between MAPK and Hippo pathway members in melanoma (A) Immunoprecipitation of RAF-1 and MST-2 in HS695T cells. (B) Western blotting showing the efficacy and specificity of RAF-1 knockdown. Protein levels were normalized to GAPDH. (C) The expression levels of MST-2, YAP and TAZ after RAF-1 knockdown, normalized to GAPDH. RAF-1, fibrosarcoma-1 proto-oncogene, serine/threonine kinase; MST-2, serine/threonine kinase 3.

cells expressed the highest levels of RAF-1. The expression levels were not significantly different among the proteins and the four cell types $(\mathrm{P}>0.05)$.

Relationship between Hippo and MAPK pathway members in melanoma. The HS695T cells expressed the highest levels of RAF-1, thus, the subsequent experiments were carried out in these cells. To determine whether there was a causal relationship between the Hippo and MAPK pathways, we performed immunoprecipitation in HS695T cells using RAF-1 as bait. The immunoprecipitation experiments revealed that RAF-1 was highly enriched with MST-2, indicating that RAF-1 bound to MST-2 in melanoma cells (Fig. 2A). Therefore, the interaction between RAF-1 and MST-2 may provide a link between the MAPK and Hippo pathways in these cells. Furthermore, we performed siRNA-mediated RAF-1 knockdown in melanoma cells. As shown in Fig. 2B, the expression of RAF-1 decreased significantly after siRNA transfection. Additionally, as presented in Fig. 2C, the expression levels of YAP and TAZ also decreased significantly after RAF-1 knockdown. However, the expression of MST-2 did not significantly change, which further suggests that RAF-1 bound to MST-2 (Fig. 2C). 

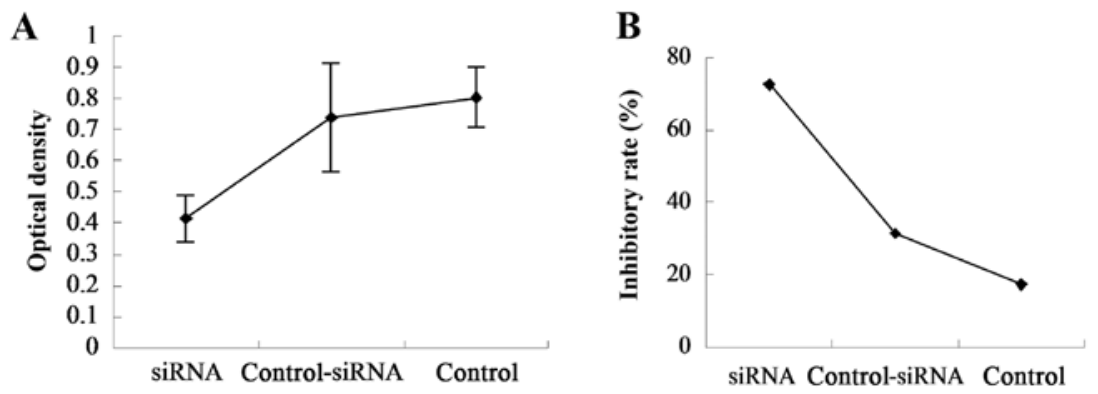

Figure 3. Cell viability assay after RAF-1 knockdown. (A) The OD of HS695T cells after $48 \mathrm{~h}$ of RAF-1 knockdown. (B) The IR of HS695T cells $48 \mathrm{~h}$ after RAF-1 knockdown. RAF-1, fibrosarcoma-1 proto-oncogene, serine/threonine kinase; OD, optical density; IR, inhibitory rate.

A

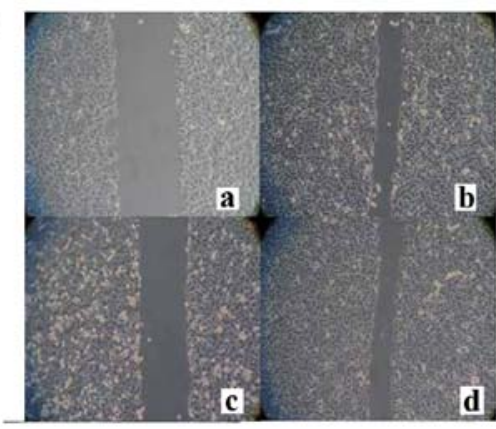

B

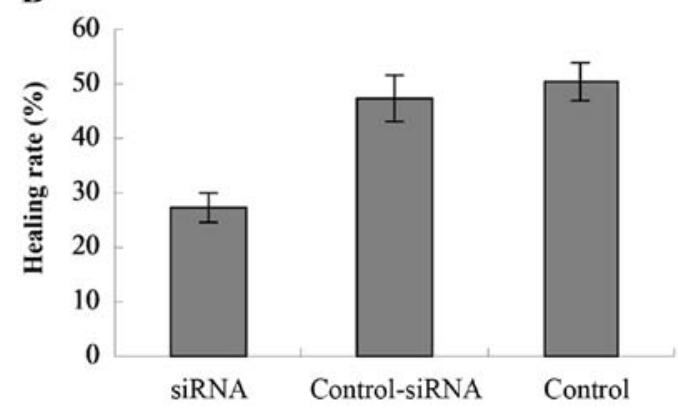

Figure 4. Cell migration assay after RAF-1 knockdown. (A) Images of cell migration assay after RAF-1 knockdown: a, image of the scratch at 0 h; images of the scratch at $48 \mathrm{~h}$ in the b, control-siRNA group; c, RAF-1-siRNA group; and d, no-siRNA control group. (B) HR in HS695T cells after 48 h of RAF-1 knockdown. RAF-1, fibrosarcoma-1 proto-oncogene, serine/threonine kinase; HR, healing rate.

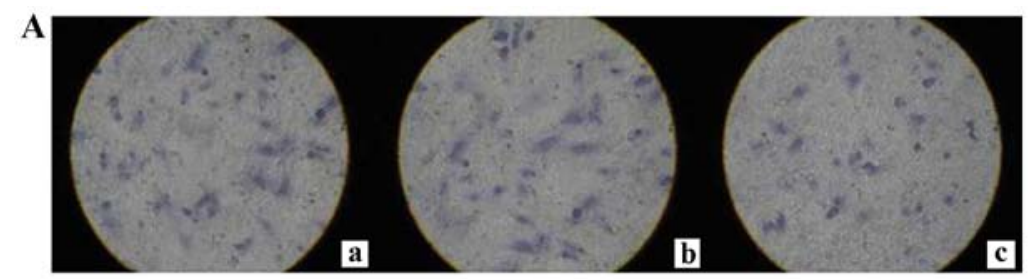

B

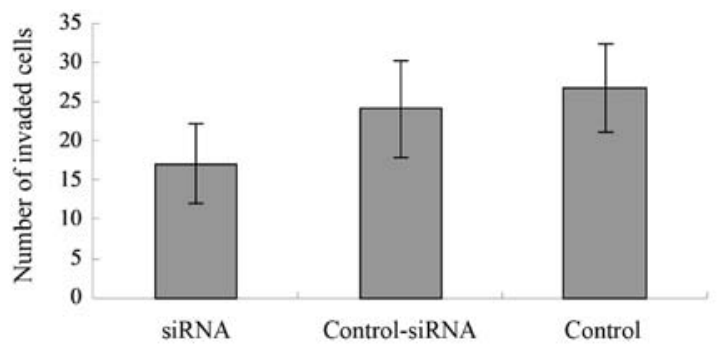

Figure 5. Cell invasion assay after RAF-1 knockdown. (A) Images of cell invasion after RAF-1 knockdown for the: a, no-siRNA control; b, control-siRNA; and c, RAF-1-siRNA group. (B) The number of invaded cells after $48 \mathrm{~h}$ of RAF-1 knockdown in HS695T cells. RAF-1, fibrosarcoma-1 proto-oncogene, serine/threonine kinase.

Effect of RAF-1 knockdown on melanoma cell viability, migration and invasion. To determine whether the RAF-1/MST-2 complex contributed to the tumorigenicity of melanoma, we performed MTT, wound-healing and cell invasion assays. The MTT assay was used to determine cell viability after $48 \mathrm{~h}$ of RAF-1 knockdown. The ODs of the RAF-1-siRNA, control-siRNA and no-siRNA control groups were $0.4157 \pm 0.0763,0.7407 \pm 0.1734$ and $0.8053 \pm 0.0991$, respectively (Fig. 3A), and their IRs were 72.56, 31.42 and $17.23 \%$, respectively (Fig. 3B). Significant differences were found among the three groups $(\mathrm{P}=0.000)$, indicating that RAF-1 knockdown significantly inhibited cell viability.

Additionally, wound-healing assay was carried out to determine changes in cell migration after RAF-1 knockdown. As shown in Fig. 4A, the number of migrated cells was significantly decreased in the RAF-1-siRNA group, but significantly increased in the control-siRNA and no-siRNA control groups. The HRs of the RAF-1-siRNA, control-siRNA, and no-siRNA control groups were $27.33 \pm 2.80,47.30 \pm 4.26$ and $50.47 \pm 3.38 \%$, respectively (Fig. 4B), and were significantly 

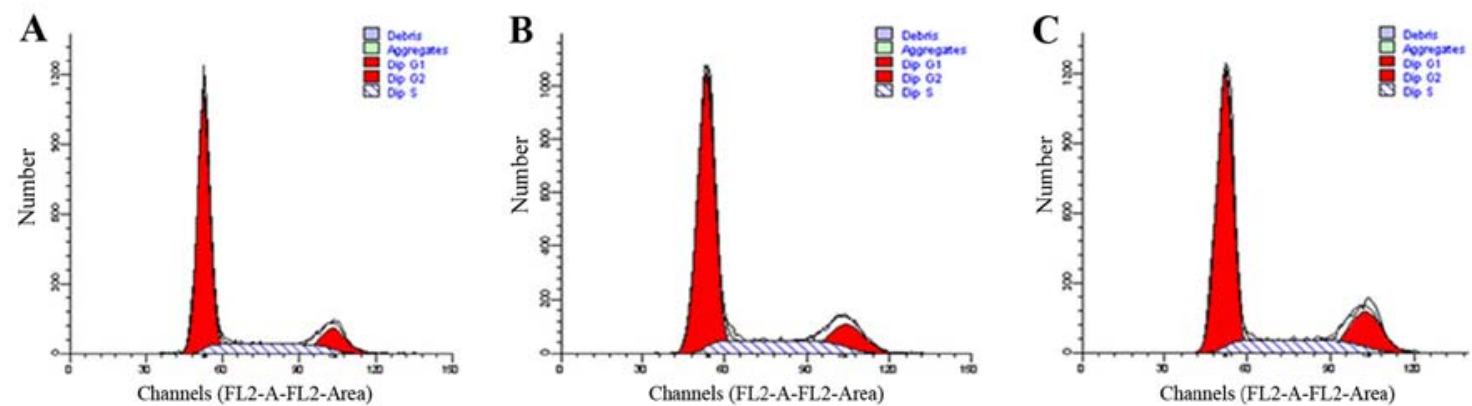

Figure 6. Cell cycle assay after $48 \mathrm{~h}$ of RAF-1 knockdown. Images of the cell cycle after $48 \mathrm{~h}$ of RAF-1 knockdown for the: (A) no-siRNA control; (B) control-siRNA; and (C) RAF-1-siRNA group. RAF-1, fibrosarcoma-1 proto-oncogene, serine/threonine kinase.

Table I. Cell cycle of HS695T cells after 48 h of RAF-1 knockdown.

\begin{tabular}{lccccr}
\hline Cell cycle & Control group $(\%)$ & Control-siRNA $(\%)$ & RAF-1-siRNA $(\%)$ & F-value & P-value \\
\hline G1 & $65.82 \pm 1.02$ & $65.90 \pm 2.64$ & $64.31 \pm 4.50$ & 0.256 & 0.783 \\
G2 & $14.30 \pm 1.18$ & $14.59 \pm 2.84$ & $15.56 \pm 4.40$ & 0.135 & 0.877 \\
S & $19.85 \pm 0.50$ & $19.50 \pm 0.41$ & $20.13 \pm 0.13$ & 2.000 & 0.216 \\
\hline
\end{tabular}
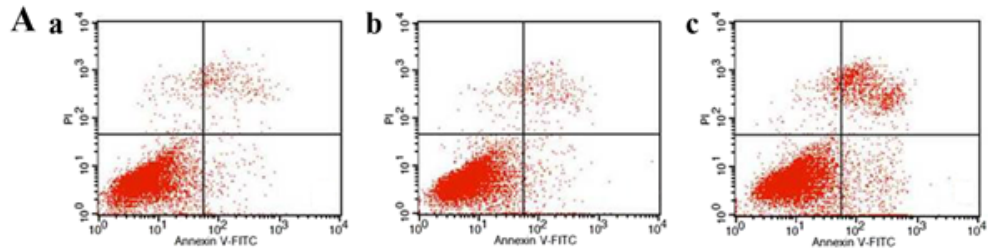

B

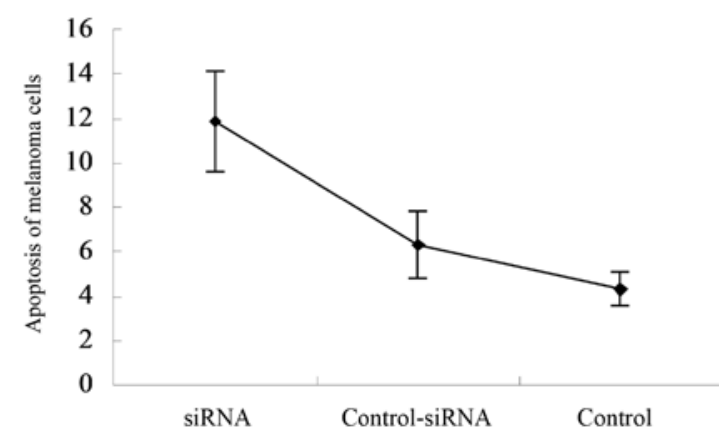

Figure 7. Apoptosis assay after $48 \mathrm{~h}$ of RAF-1 knockdown. (A) Images of cell apoptosis after $48 \mathrm{~h}$ of RAF-1 knockdown for the: a, no-siRNA control; b, control-siRNA; and c, RAF-1-siRNA group. (B) The rate of apoptosis of HS695T cells after $48 \mathrm{~h}$ of RAF-1 knockdown. RAF-1, fibrosarcoma-1 protooncogene, serine/threonine kinase.

different $(\mathrm{P}=0.000)$. These results indicate that disruption of the RAF-1/MST-2 interaction inhibited cell migration.

The Transwell cell culture chambers were used to determine the effects of RAF-1 knockdown on cell invasion (Fig. 5A). The number of invaded cells for the RAF-1-siRNA, control-siRNA, and no-siRNA control groups were 17.1 $\pm 5.1,24.1 \pm 6.2$ and $26.8 \pm 5.6$, respectively (Fig. 5B). There was a significant difference between the RAF-1-siRNA and control groups $(\mathrm{P}=0.001)$. These findings indicate that RAF-1 knockdown significantly inhibited cell invasion.

Effect of RAF-1 knockdown on the cell cycle and apoptosis of melanoma cells. To determine whether RAF-1 knockdown affected the cell cycle in melanoma cells, we performed flow cytometry after $48 \mathrm{~h}$ of RAF-1 knockdown. The results are shown in Fig. 6 and Table I. The percentages of cells in the G1 phase in the RAF-1-siRNA, control-siRNA, and no-siRNA control groups were $64.31 \pm 4.50,65.90 \pm 2.64$ and $65.82 \pm 1.02 \%$, respectively, and the difference was not statistically significant $(\mathrm{P}=0.783)$. Similarly, for cells in the $\mathrm{G} 2$ and $\mathrm{S}$ phases, no significant difference was found among the three groups.

The apoptosis of melanoma cells $48 \mathrm{~h}$ after siRNA transfection was also detected using flow cytometry. As shown in Fig. 7A and B, the apoptosis rates of melanoma cells were $11.88 \pm 2.25,6.32 \pm 1.54$ and $4.30 \pm 0.75$ for the RAF-1-siRNA, control-siRNA, and no-siRNA control groups, respectively. Significant differences were found between the control-siRNA and RAF-1-siRNA groups $(\mathrm{P}=0.006)$, and between the 
no-siRNA control and RAF-1-siRNA groups $(\mathrm{P}=0.003)$. These findings indicate that RAF-1 knockdown significantly increased the apoptosis rate of melanoma cells.

\section{Discussion}

The present study, investigated the interaction between MAPK and Hippo signaling pathway members (RAF-1, MST-2, YAP and TAZ) in four malignant melanoma cell lines. The results revealed that RAF-1, MST-2, YAP and TAZ were expressed in all the melanoma cells examined. However, RAF-1 and MST-2 were found to interact in HS695T cells. Furthermore, preventing the formation of the RAF-1/MST-2 complex (through knockdown of RAF-1) inhibited melanoma cell viability, migration and invasion, and promoted apoptosis. Our findings suggest that in malignant melanoma, the RAF-1/MST-2 interaction may provide a functional link between the MAPK and Hippo signaling pathways.

Activation of the MAPK signaling pathway, mainly owing to oncogenic mutations in proto-oncogenes such as RAF-1, plays a critical role in the promotion of melanoma cell proliferation (26). The main function of RAF-1 is to activate mitogen-activated protein/extracellular signal-regulated kinase (ERK) kinase (MEK, a component of the MAPK cascade) by direct phosphorylation (27). Additionally, disruption of RAF-1 leads to widespread apoptosis (28). Notably, this additional function (i.e., controlling apoptosis) of RAF-1 is independent of MAPK and is achieved by binding to MST-2.

MST-2 has been found to interact with both wild-type and kinase-inactive RAF-1 by mass spectrometry analysis (29). It has been demonstrated that RAF-1 controls MST-2 activity by (29): interfering with MST-2 dimerization, thus, permitting the transphosphorylation of critical residues required for MST-2 activation (31), in addition to recruiting a phosphatase to dephosphorylate these residues. The MST-2 interaction domain is located between amino acids 150 and 303 in the RAF-1 regulatory domain (32). Dissociation of the RAF-1/MST-2 complex is associated with MST-2 activation, and subsequent apoptosis. In a review study by Kyriakis (28), it has been suggested that MST-2 promotes death signaling, rather than inhibiting survival signaling, which is not consistent with the present study. In the present study, RAF-1 was found to bind MST-2 in melanoma cells. The suppression of RAF-1 and subsequent prevention of the RAF-1/MST-2 complex formation not only promoted apoptosis, but also inhibited melanoma cell proliferation significantly, which is in accordance with the findings of a recent study by Romano et al (30).

Given that RAF-1 is an effector of the MAPK signaling pathway and MST-2 is a core component of the Hippo pathway, we propose that the RAF-1/MST-2 interaction may provide a link between these two pathways. Notably, Romano et al (33) have reported that RAF-1 mutation stimulates both the Hippo and MAPK pathways, simultaneously driving apoptosis and proliferation, whereas concomitant MST-2 downregulation switches signaling to cell proliferation, transformation and survival.

The Hippo pathway plays a tumor-suppressor role and is mutated in a variety of cancers (21). This pathway is comprised of a cascade of kinases, including MST-2, YAP and TAZ. MST-2 is an upstream regulator of this pathway, while YAP and its paralog TAZ are the downstream effectors (23). A recent study found that YAP and TAZ contribute to the metastatic and invasive capacities of melanoma cells (23). Lamar et al (34) have demonstrated that YAP overexpression, with exclusive nuclear localization, improves the metastatic potential of melanoma cells. Based on the results of the present study, we postulate that RAF-1 binding may inactivate MST-2, consequently inactivating the Hippo pathway, and resulting in the accumulation of YAP and TAZ. The accumulation of YAP and TAZ may subsequently activate the target genes involved in cell proliferation and invasion. Upon knockdown of RAF-1, MST-2 could be released and activated, leading to the activation of the Hippo pathway. Yu et al (35) recently found that the Hippo pathway inhibited the expression of YAP and TAZ to regulate cell proliferation, apoptosis and differentiation, thus, modulating tissue growth and homeostasis. We, therefore, speculate that the downregulation of YAP and TAZ in the present study may be attributed to the activation of the Hippo pathway. In turn, the downregulation of YAP and TAZ may be associated with the inhibition of melanoma cell invasion and metastasis. Collectively, our data provides novel evidence suggesting that endogenous RAF-1/MST-2 interaction contributes to the deregulation of the Hippo pathway and results in the metastatic behavior of melanoma cells. Moreover, the results further reveal an intricate connection between the MAPK and Hippo pathway effector molecules.

In conclusion, our results indicate that the RAF-1/MST-2 interaction may be a novel link between the MAPK and Hippo pathways. Targeting this interaction may serve as a novel approach in the treatment of melanoma. However, further studies are needed to elucidate the mechanisms underlying the crosstalk between the two pathways.

\section{Acknowledgements}

The present study was supported by a special fund for medical service from the Jilin Finance Department (SCZSY201507).

\section{References}

1. Stewart BW and Wild C: International Agency for Research on Cancer. World Health Organization (ed). Lyon, France. International Agency for Research on Cancer. p630, 2014.

2. Bataille V, Winnett A, Sasieni P, Newton Bishop JA and Cuzick J: Exposure to the sun and sunbeds and the risk of cutaneous melanoma in the UK: A case-control study. Eur J Cancer 40: 429-435, 2004.

3. Balch CM, Gershenwald JE, Soong SJ, Thompson JF, Atkins MB, Byrd DR, Buzaid AC, Cochran AJ, Coit DG, Ding S, et al: Final version of 2009 AJCC melanoma staging and classification. J Clin Oncol 27: 6199-6206, 2009.

4. Gray-Schopfer V, Wellbrock C and Marais R: Melanoma biology and new targeted therapy. Nature 445: 851-857, 2007.

5. La Porta CA: Drug resistance in melanoma: New perspectives. Curr Med Chem 14: 387-391, 2007.

6. Todd JR, Scurr LL, Becker TM, Kefford RF and Rizos H: The MAPK pathway functions as a redundant survival signal that reinforces the PI3K cascade in c-Kit mutant melanoma. Oncogene 33: 236-245, 2014.

7. Greaves WO, Verma S, Patel KP, Davies MA, Barkoh BA, Galbincea JM, Yao H, Lazar AJ, Aldape KD, Medeiros LJ, et al: Frequency and spectrum of $B R A F$ mutations in a retrospective, single-institution study of 1112 cases of melanoma. J Mol Diagn 15: 220-226, 2013. 
8. Flaherty KT, Infante JR, Daud A, Gonzalez R, Kefford RF, Sosman J, Hamid O, Schuchter L, Cebon J, Ibrahim N, et al: Combined BRAF and MEK inhibition in melanoma with BRAF V600 mutations. N Engl J Med 367: 1694-1703, 2012.

9. Davies H, Bignell GR, Cox C, Stephens P, Edkins S, Clegg S, Teague J, Woffendin H, Garnett MJ, Bottomley W, et al: Mutations of the BRAF gene in human cancer. Nature 417: 949-954, 2002.

10. Viola JR, Rafael DF, Wagner E, Besch R and Ogris M: Gene therapy for advanced melanoma: Selective targeting and therapeutic nucleic acids. J Drug Deliv 2013: 897348-897348, 2013.

11. Su F, Viros A, Milagre C, Trunzer K, Bollag G, Spleiss O Reis-Filho JS, Kong X, Koya RC, Flaherty KT, et al: RAS mutations in cutaneous squamous-cell carcinomas in patients treated with BRAF inhibitors. N Engl J Med 366: 207-215, 2012.

12. Oberholzer PA, Kee D, Dziunycz P, Sucker A, Kamsukom N, Jones R, Roden C, Chalk CJ, Ardlie K, Palescandolo E, et al: $R A S$ mutations are associated with the development of cutaneous squamous cell tumors in patients treated with RAF inhibitors. J Clin Oncol 30: 316-321, 2012.

13. Baccarini M: An old kinase on a new path: Raf and apoptosis. Cell Death Differ 9: 783-785, 2002.

14. Antony R, Emery CM, Sawyer AM and Garraway LA: C-RAF mutations confer resistance to RAF inhibitors. Cancer Res 73 : 4840-4851, 2013.

15. Avruch J, Khokhlatchev A, Kyriakis JM, Luo Z, Tzivion G, Vavvas D and Zhang XF: Ras activation of the Raf kinase: Tyrosine kinase recruitment of the MAP kinase cascade. Recent Prog Horm Res 56: 127-155, 2001.

16. Hüser M, Luckett J, Chiloeches A, Mercer K, Iwobi M, Giblett S, Sun XM, Brown J, Marais R and Pritchard C: MEK kinase activity is not necessary for Raf- 1 function. EMBO J 20 : 1940-1951, 2001.

17. Avruch J, Zhou D, Fitamant J, Bardeesy N, Mou F and Barrufet LR: Protein kinases of the Hippo pathway: Regulation and substrates. Semin Cell Dev Biol 23: 770-784, 2012.

18. O'Neill E, Rushworth L, Baccarini M and Kolch W: Role of the kinase MST2 in suppression of apoptosis by the proto-oncogene product Raf-1. Science 306: 2267-2270, 2004.

19. Thomas NE, Edmiston SN, Alexander A, Millikan RC, Groben PA, Hao H, Tolbert D, Berwick M, Busam K, Begg CB, et al: Number of nevi and early-life ambient UV exposure are associated with $B R A F$-mutant melanoma. Cancer Epidemiol Biomarkers Prev 16: 991-997, 2007.

20. Praskova M, Xia F and Avruch J: MOBKL1A/MOBKL1B phosphorylation by MST1 and MST2 inhibits cell proliferation. Curr Biol 18: 311-321, 2008.

21. Harvey KF, Zhang X and Thomas DM: The Hippo pathway and human cancer. Nat Rev Cancer 13: 246-257, 2013.
22. Pollock PM, Harper UL, Hansen KS, Yudt LM, Stark M, Robbins CM, Moses TY, Hostetter G, Wagner U, Kakareka J, et al: High frequency of $B R A F$ mutations in nevi. Nat Genet 33: 19-20, 2003.

23. Nallet-Staub F, Marsaud V, Li L, Gilbert C, Dodier S, Bataille V, Sudol M, Herlyn M and Mauviel A: Pro-invasive activity of the Hippo pathway effectors YAP and TAZ in cutaneous melanoma. J Invest Dermatol 134: 123-132, 2014.

24. Lin L and Bivona TG: The Hippo effector YAP regulates the response of cancer cells to MAPK pathway inhibitors. Mol Cell Oncol 3: e1021441, 2015.

25. Zheng HX, Wu LN, Xiao H, Du Q and Liang JF: Inhibitory effects of dobutamine on human gastric adenocarcinoma. World J Gastroenterol 20: 17092-17099, 2014.

26. Chin L, Garraway LA and Fisher DE: Malignant melanoma: Genetics and therapeutics in the genomic era. Genes Dev 20: 2149-2182, 2006.

27. Hüser M, Luckett J, Chiloeches A, Mercer K, Iwobi M, Giblett S, Sun XM, Brown J, Marais R and Pritchard C: MEK kinase activity is not necessary for Raf-1 function. EMBO J 20: 1940-1951, 2001.

28. Kyriakis JM: The integration of signaling by multiprotein complexes containing Raf kinases. Biochim Biophys Acta 1773: 1238-1247, 2007.

29. O'Neill E, Rushworth L, Baccarini M and Kolch W: Role of the kinase MST2 in suppression of apoptosis by the proto-oncogene product Raf-1. Science 306: 2267-2270, 2004.

30. Romano D, Matallanas D, Weitsman G, Preisinger C, Ng T and Kolch W: Proapoptotic kinase MST2 coordinates signaling crosstalk between RASSF1A, Raf-1, and Akt. Cancer Res 70: 1195-1203, 2010

31. Hay BA and Guo M: Coupling cell growth, proliferation, and death. Hippo weighs in. Dev Cell 5: 361-363, 2003.

32. Avruch J, Zhou D, Fitamant J, Bardeesy N, Mou F and Barrufet LR: Protein kinases of the Hippo pathway: Regulation and substrates. Semin Cell Dev Biol 23: 770-784, 2012.

33. Romano D, Nguyen LK, Matallanas D, Halasz M, Doherty C, Kholodenko BN and Kolch W: Protein interaction switches coordinate Raf-1 and MST2/Hippo signalling. Nat Cell Biol 16: 673-684, 2014

34. Lamar JM, Stern P, Liu H, Schindler JW, Jiang ZG and Hynes RO: The Hippo pathway target, YAP, promotes metastasis through its TEAD-interaction domain. Proc Natl Acad Sci USA 109: E2441-E2450, 2012

35. Yu FX, Meng Z, Plouffe SW and Guan KL: Hippo pathway regulation of gastrointestinal tissues. Annu Rev Physiol 77: 201-227, 2015. 